\title{
A case of omenn syndrome accompanied by bilateral cystine stones: case report
}

\begin{abstract}
Omenn syndrome is one type of combined immunodeficiency, characterized with hepatosplenomegaly, lymphadenopathy, and recurrent infections and has an autosomal recessive pattern of inheritance. $\mathrm{T}$ lymphocyte level can be normal in peripheral blood but their function is impaired. B lymphocyte level is very low to none. Cystinuria is renal re-absorption defect of dibasic amino acids, inherited autosomal recessive. In literature, Omenn Syndrome and cysteine stone togetherness is not found, so that our case is very interesting.

Five months old girl was applied Immunology Department with skin eruption. There was no family history for immune deficiency and not consanguineous marriage between mother and father. Physical examination was showed, exfoliative erythroderma and hepatomegaly. Laboratory results: leukocyte count $6540 / \mathrm{mm}^{3}$,absolute neutrophil count $2270 / \mathrm{mm}^{3}$, absolute lymphocyte count $1560 / \mathrm{mm}^{3}$, absolute eosinophil count $2220 / \mathrm{mm}^{3}$, serum IgG level $171 \mathrm{mg} / \mathrm{dl}$, IgA level 5,81mg/dl, IgM level 24,5mg/dl, IgE level $1270 \mathrm{mg} /$ $\mathrm{dl}$ were found. T lymphocyte count $1092 / \mathrm{mm}^{3}$, B lymphocyte count $6 / \mathrm{mm}^{3}$, NK count 332/ $\mathrm{mm}^{3}$ was found.

There was not found appropriate donor for patient and so planned haploidentical bone marrow transplantation. In preparation of bone marrow transplantation, abdominal contrast tomography was showed bilateral kidney stones. In lab tests, cystinuria was detected in urine and thought to be bilateral cysteine stone; simultaneous bilateral percutaneous nephrolithotomy operation was performed. Stone analysis resulted as cysteine stone.

Autosomal recessive two different diseases are found together is very interesting condition. Challenging incident that can be caused by a reason or it can be only coincidence? In Omenn Syndrome, cysteine and tyrosine amino acids sequencing alteration is known and can it be the reason of cysteine stone formation?
\end{abstract}

Keywords: Omenn syndrome; Percutaneous nephrolithotomy; Cystine stone
Volume 6 Issue I - 2018

\author{
Murat Cansever,' Ayse Seda Pinarbasi, ${ }^{2}$ \\ Numan Baydilli, ${ }^{3}$ Musa Karakukcu, ${ }^{4}$ Turkan \\ Patiroglu' \\ 'Department of Pediatrics, Division of Immunology, Erciyes \\ University, Turkey \\ ${ }^{2}$ Department of Pediatrics, Division of Nephrology, Erciyes \\ University, Turkey \\ ${ }^{3}$ Department of Urology, Erciyes University, Turkey \\ ${ }^{4}$ Department of Pediatrics, Division of Hematology and \\ Oncology, Erciyes University, Turkey
}

Correspondence: Numan Baydilli, Faculty of Medicine, Department of Urology, Erciyes University, 38034, Kayseri, Turkey, Tel 9053578602 65, Fax 903524377613

Email dr_numan38@hotmail.com

Received: January 22, 2018 | Published: February 05, 2018
Abbreviations: OS, omenn syndrome; BMT, bone marrow transplantation; Mini-PNL, mini-percutaneous nephrolithotomy; RAG-I, recombination activating gene- I; ANC, absolute neutrophil count; ALC, absolute lymphocyte count; WBC, white blood cell; Hgb: hemoglobin

\section{Introduction}

Omenn Syndrome (OS) is a rare autosomal recessive form of severe combined immunodeficiency characterized by failure to thrive, erythroderma, persistent diarrhea, lymphadenopathy, hepatosplenomegaly; severe recurrent infections. ${ }^{1}$ The syndrome may be accompanied by normal or elevated levels of IgE, lymphocytosis, eosinophilia and hypogammaglobinemia. ${ }^{1}$ The syndrome, also known as familial reticuloendotheliosis, was first described by Gilbert Omenn $^{1}$ in 1965 . The estimated incidence is $1 / 50000-1 / 75000$, with equal frequency in both genders.

Cystine stones are the most common among inherited disorders characterized by urinary stone formation. In children, $4-8 \%$ of all urinary system stones are cystine stones. The estimated worldwide prevalence is $1 / 7000 .^{2}$

In literature, several anomalies and pathologies associated with various forms of immune deficiency have been reported; however, clinical entities associated with Omenn syndrome were not mentioned. In this paper, the association of bilateral cystine stones with Omenn
Syndrome, a rare form of immune deficiency, was reported. Written informed consent was obtained from the parents of the patient.

\section{Case presentation}

A female infant born to a second gravida 38 year-old mother at 38 weeks gestation by Cesarian section with a birth weight of 3050 $\mathrm{g}$ was found normal on initial physical examination. The mother and father had no reported blood relation although they were from the same town. First child was a healthy 5 year-old girl. There was no family history of an immune deficiency disorder. The patient had no complaints until 2 months postpartum when she presented with impaired sucking, hypotonia, generalized exfoliative erythroderma and upon detection of lymphopenia she was hospitalized for further investigation and treatment.

Physical examination revealed the following: weight: $4400 \mathrm{~g}(10-$ 25 p), height: $55.5 \mathrm{~cm}(10-25 \mathrm{p})$, head circumference: $38 \mathrm{~cm}(10-25$ p) pulse rate: $116 / \mathrm{min}$., respiration rate: $40 / \mathrm{min}$, anterior fontanel $3 \times 2$ $\mathrm{cm}$, posterior fontanel $0.5 \times 0.5 \mathrm{~cm}$, alopecia, erythroderma on scalp and generalized exfoliation (Figure 1A \& B). Overall status was well, generalized exfoliative dermatitis and erythroderma were detected. Auscultation of lungs and the cardiovascular system were normal. The abdomen was soft and non-tender, the liver was palpable $2-3 \mathrm{~cm}$ under the costal margin. Spleen was non-palpable and other systems were normal on examination. 
Laboratory findings were as follows White Blood Cell: $6540 / \mathrm{mm}^{3}$, Red Blood Cell:4.890.000/mm, ${ }^{3}$ Hemoglobin: 12g/dl, Hematocrit: $\% 35$, platelets: $592.000 / \mathrm{mm}^{3}$. Peripheral smear showed $34 \%$ polymorph nuclear leucocytes, $24 \%$ lymphocytes, $4 \%$ monocytes, $41 \%$ eosinophils, clusters of four-five or seven-eight platelets in microscopic areas evaluated. Values of blood gases, blood sugar, hepatic and renal function tests, and blood electrolytes were within normal range, PA lung x-ray was normal, thymus zone was visible.

Following collecting samples for blood and urine cultures, dual wide-spectrum antibiotic therapy and prophylactic antifungal therapy was initiated based on disintegrated skin and possible underlying immune deficiency. Expholiative dermatitis was treated with $50 \%$ "Eau de Rose" and 50\% glycerine cream, Liniment oleo calcaire and permasolwere applied on scalp lesions.

Physical examination findings and blood values suggested the diagnosis of Omenn Syndrome and immunoglobulin evaluation revealed the following results; Ig M: $24.5 \mathrm{mg} / \mathrm{dl}$, Ig G: $171 \mathrm{mg} / \mathrm{dl}$, Ig A: $5.81 \mathrm{mg} / \mathrm{dl}$, Ig E: $1270 \mathrm{mg} / 1$. Lymphocyte panel of the patient is presented in (Table 1). Echocardiography and cranial ultrasound examinations showed normal findings. Bilateral multiple renal stones were observed on abdominal ultrasound and tomography (Figure 1C).

On lymphocyte panel, CD3 was normal and activated Tlymphocytes were increased. Total B lymphocyte count was scarce. Activated T cells were non-functional. Physical examination findings exfoliative dermatitis, erythroderma, alopecia, laboratory findings eosinophilia, hypogammaglobinemia, increased immunoglobulin E levels, absence of total B cells, increased activated $\mathrm{T}$ cell count suggested the patient had Omenn Syndrome Blood sample for genetic analysis was sent abroad. Treatment with $0.5 \mathrm{~g} / \mathrm{kg}$ intravenous immunoglobulin every 3 weeks was initiated.

Bone marrow transplantation (BMT) was planned for curative treatment. However, nephrology council recommended transplantation can be performed only after appropriate management of multiple stones. Pediatric Urology Department performed lithotripsy using Holmium laser, followed by bilateral mini-percutaneous nephrolithotomy (mini-PNL) operation. The operation was completed without any problems; nephrostomy catheters were removed at postoperative 24 hours (Figure 1D). Stones were sent for analysis; results showed they were cystine stones (Figure 1E). Transplantation was not recommended before 15 days post-operation. During this period, as part of routine tests for transplantation, toxoplasmosis was found positive and treatment was initiated. Cerebrospinal fluid specimen was also found positive for toxoplasma, therefore treatment was continued until negative results were obtained from both plasma and cerebrospinal fluid. The patient did not have a haploidentical sibling, thus haploidentical BMT from mother or father was planned. Both parents had normal immunoglobulin values and lymphocyte panels. Following tissue typing of parents, the father was identified as the bone marrow donor.

\section{Discussion}

Omenn Syndrome is a rare form of combined immune deficiency, first described by Gilbert Omenn ${ }^{3}$ in 1965 in the child of related parents. Up to date, at least 67 cases have been reported ${ }^{1}$. Villa et al. ${ }^{4}$ have demonstrated RAG- 1 and RAG-2 mutations in OS and thus specified the pathology in precursor $\mathrm{B}$ and $\mathrm{T}$ cells. The presenting finding is erythematous rash in $85 \%$, which was observed in $98 \%$ of the reported cases. Age of symptom onset is first two weeks of life in approximately half of cases. Other findings may be present at onset or may occur later. Erythroderma, which is a preceding symptom, should alert physicians for immunological evaluation if coexists with hepatosplenomegaly, lymphadenopathy.

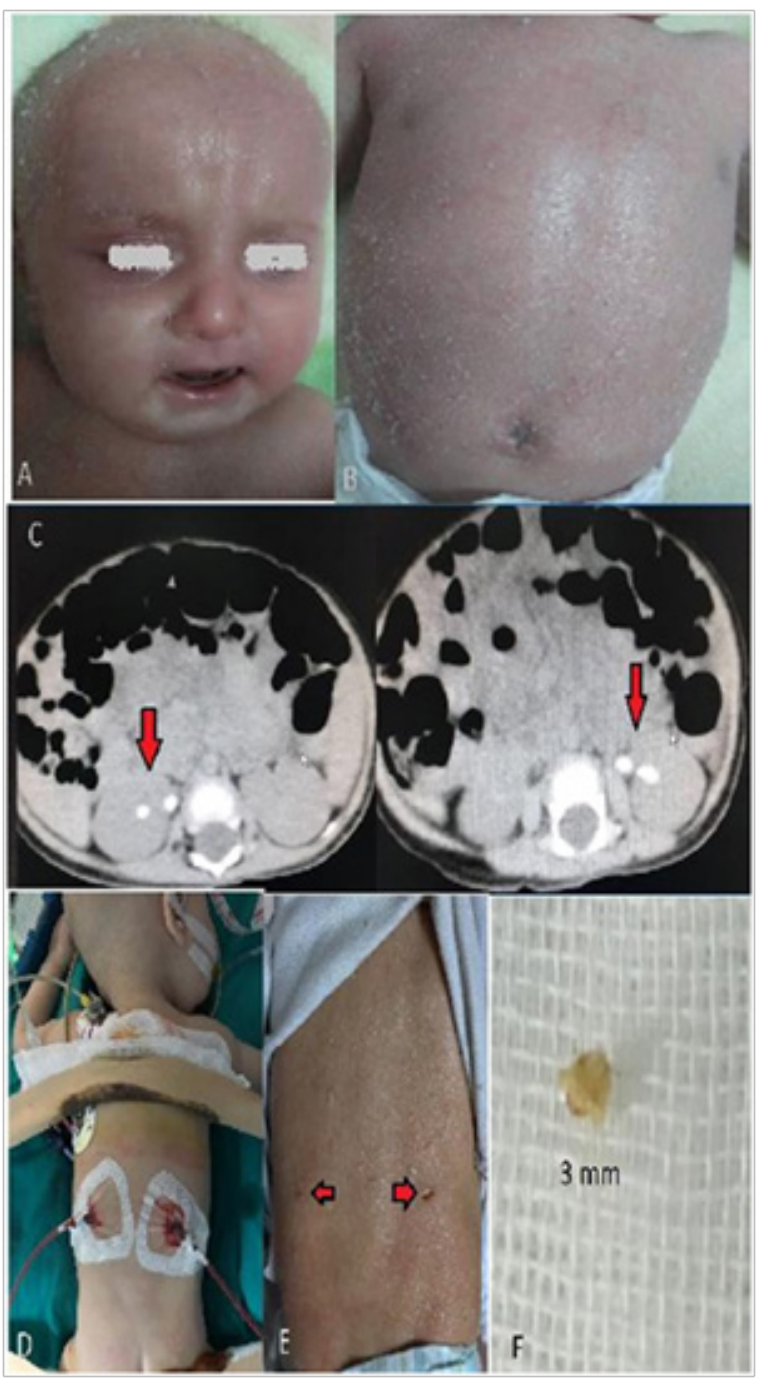

Figure I (a) Micrognathia and exfoliative erythrodermic rashes in face and hairy skin (b) Exfoliative erythrodermic rashes in body, (c) Right kidney (4,7 $\mathrm{mm}$ and 4,6 $\mathrm{mm}$ ) and left kidney $(7,5 \mathrm{~mm}$ and $6,7 \mathrm{~mm})$ stones in abdomen CT image, (d) $10 \mathrm{~F}$ bilateral nephrostomy tubes images after Mini PNL operation, (e) Image of operation area after I week of operation (f) Stone sample (cystine stone) which was taken for stone analysis during operation.

Other physical examination findings include hepatosplenomegaly $(88 \%)$, lymphadenopathy $(80 \%)$, recurrent infections $(72 \%)$, and alopecia $(57 \%){ }^{1}$

In our case, erythroderma and exfoliative skin lesions which occurred at 2 months of age were complicated by hepatomegaly. The laboratory findings specific for OS are eosinophilia in 55\% of cases and leukocytosis secondary to lymphocytosis. Our patient had normal leucocyte count but eosinophilia was present. Hypogammaglobinemia may not be detected in OS cases during the period following birth due to transplacental transmission. IgE values are high. Hypogammaglobinemia is often detected during follow-up. ${ }^{1,4}$

Regarding immunoglobulin values of our patient, $\operatorname{IgM}, \operatorname{IgA}$ and IgG were found low while IgE was high. The total B cell absence and increased numbers of activated $\mathrm{T}$ cells in lymphocyte panel of patient were expected findings. 
Table I Laboratory findings of the patient

\begin{tabular}{|c|c|c|c|c|c|}
\hline \multirow{2}{*}{ Hemogram } & WBC (mm3) & Hgb (gr/dl) & Platelets $(\mathrm{mm} 3)$ & ANC (mm3) & ALC (mm3) \\
\hline & 6540 & 12 & 592000 & 2270 & 900 \\
\hline \multirow{2}{*}{ Immunglobulins } & $\operatorname{lgG}(\mathrm{mg} / \mathrm{dl})$ & $\operatorname{lgM}(\mathrm{mg} / \mathrm{dl})$ & $\lg A(m g / d l)$ & $\lg E(I U / m l)$ & Eosinofil (\%)/ (mm3) \\
\hline & 171 & 24,5 & 5,81 & 1270 & $41,7 / 2610$ \\
\hline \multirow{2}{*}{ Lymphocyte Subtype } & CD3 (\%) / (mm3) & CD4 (\%) / (mm3) & CD8 (\%) / (mm3) & CDI9 (\%) / (mm3) & CD I6-56 (NK)B (\%) / (mm3) \\
\hline & $56,8 / 5 \mathrm{II}$ & $34,6 / 311$ & $20,8 / 187$ & $0,9 / 8$ & $29,9 / 269$ \\
\hline
\end{tabular}

ANC, absolute neutrophil count;ALC, absolute lymphocyte count;WBC, white blood cell; hgb: hemoglobin

Differential diagnosis should include GVHD, atopic dermatitis, histiocytosis $\mathrm{X}$ and other immune deficiencies. ${ }^{1}$

Omenn Syndrome includes absence of B lymphocytes and $\mathrm{T}$ lymphocyte dysfunction. This is suggested to be associated with "missense" mutations of "recombination activating gene (RAG-I)", RAG-2, Artemis, IL-7R and RMRP (RNA component of mitochondrial RNA processing endoribonuclease) genes that are involved in development of B and T lymphocytes. ${ }^{5,6}$ The IL-7R gene defect in Omenn Syndrome is caused by G375T mutation of IL7R protein, which occurs as a result of translocation of thyrosine at position 118 on outer region of the protein with cysteine ${ }^{7}$. The IL-7R gene defect presents with homozygous clinical features after polymorphic changes in $5 \mathrm{p} 13$ chromosome. $^{7}$

OS is a fatal disease if untreated, curative treatments including BMT or cord blood stem cell transplantation are required. However, outcomes are poor compared to other forms of SCID. ${ }^{8}$

Although two case reports were published claiming cure was obtained with only supportive therapy or corticosteroids and epipodophyllotoxins the first case was later reported to be an "Omennlike" syndrome while maternal chimerism could not be excluded in the second case. Interferon gamma can be considered as an option to reduce $\mathrm{T}$ helper-2 immune response. ${ }^{2,9}$ Two cysteine amino acid molecules bound with a disulfide bond forms a cystine molecule. The most common mutation in the pathogenesis of cystine metabolism disorder is M467T mutation which has a prevalence of $26 \% .{ }^{10}$ Gene defects related to cystine metabolism disorders are usually located on $2 \mathrm{p}$ and $19 \mathrm{q}$ chromosomes. ${ }^{10}$

The cysteine amino acid sequence translocation on the outer region of the protein responsible for IL-7R gene defect in Omenn Syndrome and cystine metabolism disorder are localized on different chromosomes. However, abnormalities related to cysteine amino acid have been demonstrated in both IL-7R gene defect in OS and cystine metabolism disorders. ${ }^{7,10}$ Further studies are required to confirm whether the Omenn Syndrome and Cystine stone coexistence in our case is random or secondary to a shared cysteine amino acid disorder.

This case with Omenn syndrome, a rare form of combined immune deficiency, accompanied by cystine stones was reported with the aim to highlight the possible common molecular genetic mechanism, although random coexistence cannot be excluded. No previous reports are available in literature on coexistence of these two conditions.

\section{Acknowledgements}

Conception; M.C, Design; N.B, Analysis; A.S.P, Interpretation or drafting the article for critically important intellectual content: M.K, T.P

\section{Conflicts of interest}

No conflict of interest was declared by the authors.

\section{References}

1. Aleman K, Noordzij JG, de Groot R, van Dongen JJ, Hartwig NG (2001) Reviewing Omenn Syndrome. Eur J Pediatr 160: 718-725.

2. Polinsky MS, Kaiser BA, Balaurte HJ (1987) Urolithiasis in childhood. Pediatr Clin North Am 34(3): 683-710.

3. Omenn GS (1965) Familial reticuloendotheliosis with eosinophilia. N Eng J Med 273: 427-432.

4. Villa A, Santagat S, Bozzi F, Giliani S, Frattini A, et al. (1998) Partial V(D)J Recombination activity leads to Omenn Syndrome. Cell 93(5): 885-896.

5. Rego S, Kemp A, Wong M, Knight P (2006) Omenn syndrome: therapeutic effects of cyclosporin. J Paediatr Child Health 42(5): 319320

6. Geha RS, Notarangelo LD, Casanova JL, Chapel H, Conley ME, et al. (2007) Primary immunodeficiency diseases: an update from the International Union of Immunological Societies Primary Immunodeficiency Diseases Classification Committee. J Allergy Clin Immunol 120(4): 776-794.

7. Giliani S, Bonfim C, de Saint Basile G, Lanzi G, Brousse N, et al. (2006) Omenn syndrome in an infant with IL7RA gene mutation. J Pediatr 148(2): 272-274.

8. Notarangelo LD (2010) Primary immunodeficiencies. J Allergy ClinImmunol 125(2 Suppl 2): 182-194.

9. Niehues T, Perez-Becker R, Schuetz C (2010) More than just SCID thephenotypic range of combined immunodeficiencies associated with mutations in the recombinase activating genes (RAG) 1 and 2. Clin Immunol 135(2): 183-192.

10. Lahme S, Bichler KH, Eggermann T, Lang F (2002) Genomic and functional investigations of mutations of the SLC3A1 gene in cystinuria. UrolInt 69(3): 207-211. 